\section{OPEN ACCESS}

Edited by:

Olga Savvidou,

National and Kapodistrian University

of Athens, Greece

Reviewed by:

Seifollah Gholampour,

University of Chicago, United States

Tanya C. Garcia,

University of California, Davis,

United States

*Correspondence:

Xiaoqing Wang

osteoclast2006@163.com

${ }^{\dagger}$ These authors have contributed equally to this work and share first

authorship

Specialty section:

This article was submitted to

Orthopedic Surgery,

a section of the journal

Frontiers in Surgery

Received: 12 September 2021 Accepted: 20 December 2021

Published: 13 January 2022

Citation:

Liu P, Xiao JX, Zhao C, LiX, Sun G, Yang $F$ and Wang $X$ (2022) Factors Associated With the Accuracy of Depth Gauge Measurements. Front. Surg. 8:774682.

doi: 10.3389/fsurg.2021.774682

\title{
Factors Associated With the Accuracy of Depth Gauge Measurements
}

\section{Pengcheng Liu ${ }^{1 \dagger}$, Joanna Xi Xiao ${ }^{2 \dagger}$, Chen Zhao ${ }^{1 \dagger}$, Xiaodong Li $^{1}$, Guantong Sun ${ }^{1}$, Fei Yang ${ }^{1}$ and Xiaoqing Wang ${ }^{1 *}$}

${ }^{1}$ Shanghai Key Laboratory of Orthopedic Implant, Department of Orthopedics, Shanghai Ninth People's Hospital, Shanghai Jiao Tong University School of Medicine, Shanghai, China, ${ }^{2}$ School of Clinical Medicine, The National University of Ireland Galway, Galway, Ireland

Background: It is important to select appropriate screws in orthopedic surgeries, as excessively long or too short a screw may results failure of the surgeries. This study explored factors that affect the accuracy of measurements in terms of the experience of the surgeons, passage of drilled holes and different depth gauges.

Methods: Holes were drilled into fresh porcine femurs with skin in three passages, straight drilling through the metaphysis, straight drilling through the diaphysis, and angled drilling through the diaphysis. Surgeons with different surgical experiences measured the holes with the same depth gauge and using a vernier caliper as gold standard. The length of selected screws, and the time each surgeon spent were recorded. The measurement accuracy was compared based on the experiences of the surgeons and the passage of drilled holes. Further, parameters of depth gauges and 12-mm cortical bone screws from five different manufacturers were measured.

Results: A total of 13 surgeons participated in 585 measurements in this study, and each surgeon completed 45 measurements. For the surgeons in the senior, intermediate, and junior groups, the average time spent in measurements was 689, 833, and $785 \mathrm{~s}$ with an accuracy of 57.0, 42.2, and $31.5 \%$, respectively. The accuracy and measurement efficiency were significantly different among the groups of surgeons $(P<0.001)$. The accuracy of measurements was $45.1 \%$ for straight metaphyseal drilling, $43.6 \%$ for straight diaphyseal drilling, and 33.3\% for angled diaphyseal drilling ( $P=0.036)$. Parameters of depth gauges and screws varied among different manufacturers.

Conclusion: Both observer factor and objective factors could affect the accuracy of depth gauge measurement. Increased surgeon's experience was associated with improvements in the accuracy rate and measurement efficiency of drilled holes based on the depth gauge. The accuracy rate varied with hole passages, being the lowest for angled drilled holes.

Keywords: depth gauge, trauma surgery, open reduction and internal fixation, screw length, drilling 


\section{INTRODUCTION}

Bone drilling and screw selection are usually required in open reduction and internal fixation (ORIF) performed as a surgical treatment for fractures $(1,2)$. Despite previous studies about optimal drilling parameters to reach maximum accuracy (27 ), there are still concerns about rapid and accurate screw selection intraoperatively. Excessively long screws may rupture the soft tissues and tendons, causing pain and injury to blood vessels and nerves (8-14). It is reported that complications include tendonitis and tendon rupture can occur in $12-23 \%$ cases associated with excessively long screws (8). However, if the screw is too short and does not reach the trans cortex, the holding force may be insufficient, resulting in instability of the internal fixation (15). Repeated intraoperative screw replacements due to inaccurate measurements may compromise the threaded trail along the canal, reduce the holding force, possibly leading to a larger threaded "rescue" screw or even failure of the internal fixation (16-19).

Currently, depth gauge, intraoperative fluoroscopy, and preoperative CT are commonly used methods to estimate screw length. The depth gauge is an essential device used in ORIF for anatomically accurate and safe insertion of screws, being widely accepted by orthopedic surgeons. However, few studies have investigated factors that may affect the measurement accuracy of a depth gauge. The most common error is an inaccurate measurement of the length of the drilled hole, subsequently leading to inaccurate screw choice. Using a cadaveric model that mimics clinical situations of ORIF of proximal phalangeal fractures, Jernigan et al. (20) observed that most experienced surgeons were less likely to place short or excessively long screws. Besides, tactile feedback varies due to differences in cortical bone thickness and density at the metaphysis and diaphysis (21) resulting in measurement errors, which makes different passages of drilled holes another factor that influences measurement accuracy. A recent veterinary study also suggested that different depth gauges may affect the accuracy of the measurement (22).

So far, the factors that affect the accuracy of measurement of drilled holes using a depth gauge remains inconclusive. The aim of our study was to further explore factors related to the accuracy of depth gauge measurement. We hypothesized that both observer factor (such as surgeon experience) and objective factors (such as the passage of drilled holes and parameters of the depth gauges) could influence measurement accuracy. To the best of our knowledge, this study is the most comprehensive one concerning factors related to the accuracy of depth gauge measurement. Moreover, we introduce the indicator of efficiency of measurements for the first time, taking into account both the speed and accuracy rate of measurements.

\section{MATERIALS AND METHODS}

\section{Study Design}

To simulate real surgical operations, the dorsal part of three fresh porcine femurs with skin was subjected to a straight longitudinal skin incision. Soft tissues on the lateral aspect were separated, exposing the diaphysis and metaphysis of the femur.

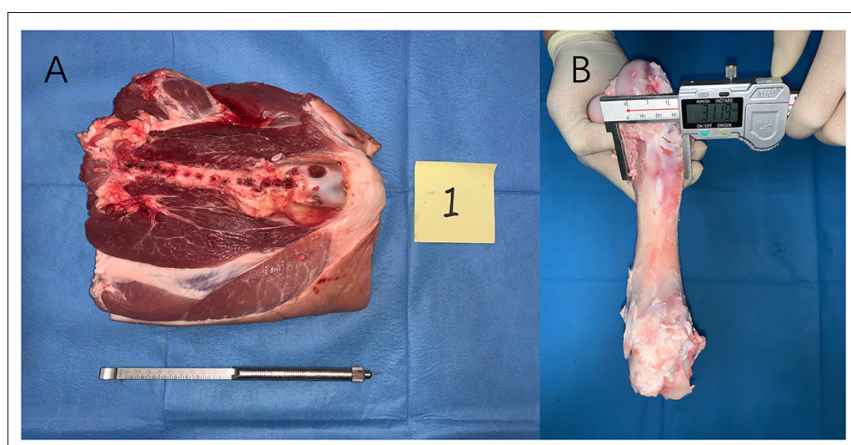

FIGURE 1 | (A) To simulate surgical operations, a dorsal part of three fresh porcine femurs with skin was accessed using a straight longitudinal skin incision. (B) After all measurements were finished the soft tissue was peeled off, and the holes were measured with a calibrated vernier caliper with $0.01 \mathrm{~mm}$ precision and scale from 0 to $150 \mathrm{~mm}(0122032,355-101$,

Hengliang, Shanghai, China); the result of which was defined as the actual length of the hole.

Holes were drilled latero-medially along the length of a bone from proximal femur to distal end using a $3.2 \mathrm{~mm}$ diameter drill bit. For each bone, holes were drilled with three different passage types in random order, namely straight drilling through the metaphysis, straight drilling through the diaphysis, and angled drilling through the diaphysis. Finally, all holes broke through the trans cortex and 45 holes were used, with 15 holes in each type of passage. 7 holes were excluded because 6 were too close to their adjacent holes, and 1 perforated the joint.

A total of 13 orthopedic trauma surgeons participated in the experiment and were divided into three groups according to their experience or training level: senior group (3 surgeons with $\geq 10$ years after medical school graduation), intermediate group (4 surgeons with 5-10 years after medical school graduation), and junior group (6 surgeons within 5 years of medical school graduation). Each surgeon was asked to use the same depth gauge with a 110-mm-long scale (319.100, Synthes) on each drilled hole, then report the screw length for the respective hole, with options consisting of integral increments of $1 \mathrm{~mm}$ (and without consideration of plate variables). In parallel, the total time each surgeon took to measure all 45 holes was recorded.

\section{Definition of Ideal Screw Length}

To determine whether the selected screw length was ideal, porcine femurs were stripped of all soft tissue, and then measured by two observers (XQW and PCL) using a calibrated vernier caliper with $0.01 \mathrm{~mm}$ precision and scale from 0 to $150 \mathrm{~mm}$ (0122032,355-101, Hengliang, Shanghai, China), the average of which was used as the actual measurement of the hole (Figure 1). In theory, an ideal screw is the smallest available size in which screw threads perforate and sustain the trans cortex. We assumed the threaded portion of the screw was equal to the length of the screw without correction for head height, which was $2 \mathrm{~mm}$ in this study. Comparing the results of the depth gauge and 


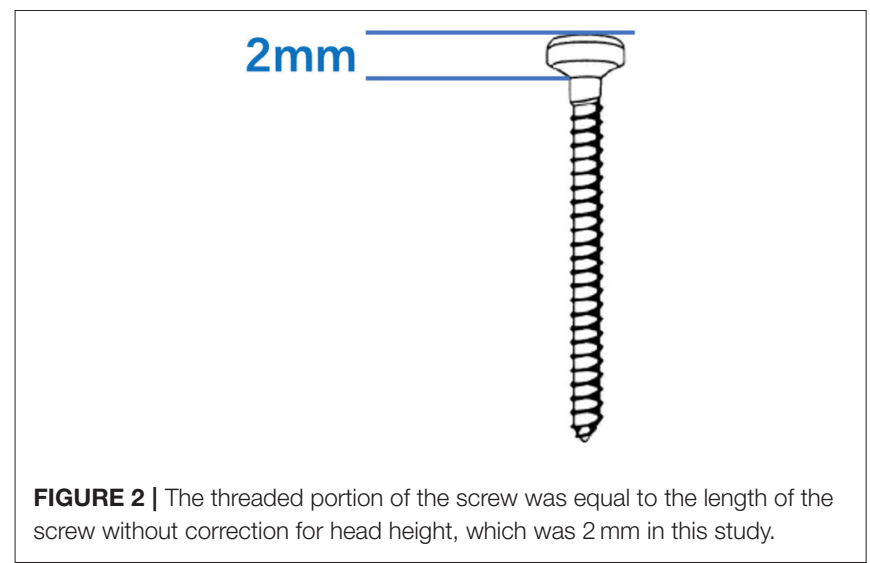

the vernier caliper, the position of the screw tip relative to the trans cortex was determined, that is, the length of the screw tip to the trans cortex $=$ (length of the selected screw-2 mm)the actual measured value of the hole (Figure 2). Ideal screws were those in which the tip of the screw reached the surface of the trans cortex but did not protrude more than $1.0 \mathrm{~mm}$ beyond it, with a positive length of the screw tip on the trans cortex. Short screws were those that failed to reach the trans cortical surface, with a negative length of the screw tip on the trans cortex.

Theoretically, sufficient holding force can be obtained when the screw reaches the ideal length of the screw. Prior biomechanical data have demonstrated that screws that extend past the volar cortical surface have higher pull-out strength than screws that do not extend past the surface 18 and that there was no marginal increase in pull-out strength for screws that extended beyond $1 \mathrm{~mm}$ past the volar surface of the bone.

The accuracy of measurements was determined as the number of screws with an ideal length divided by the total number of measurements.

The efficiency of measurements was determined as the number of screws with an ideal length divided by the total time spent by each surgeon (unit: number of screws per minute).

\section{Measurement of Different Depth Gauges and Screws}

To evaluate the influence of different depth gauges on measurement accuracy, six depth gauges from four different manufacturer (319.010, Synthes; SY 9125, Shuangyang, Jiangsu, China; SC15G002, Zimmer Biomet; KM651849, DOUBLE MEDICAL; 191003255, WEGO) (Figure 3) and five 12-mm cortical bone screws (404.812, Synthes; 0106936794823478, Shuangyang; 815037012, Zimmer Biomet; 040010022, DOUBLE MEDICAL; 6133512, WEGO) were collected for further study. Each depth gauge was set to read $12 \mathrm{~mm}$ on it's scale bar. The length of the rod (L1) and the length of the 12-mm cortical bone screws [including its full length (L2) and length of the body part (L3)] (Figure 4) were measured by one observer (XQW) using a calibrated vernier caliper with $0.01 \mathrm{~mm}$ precision

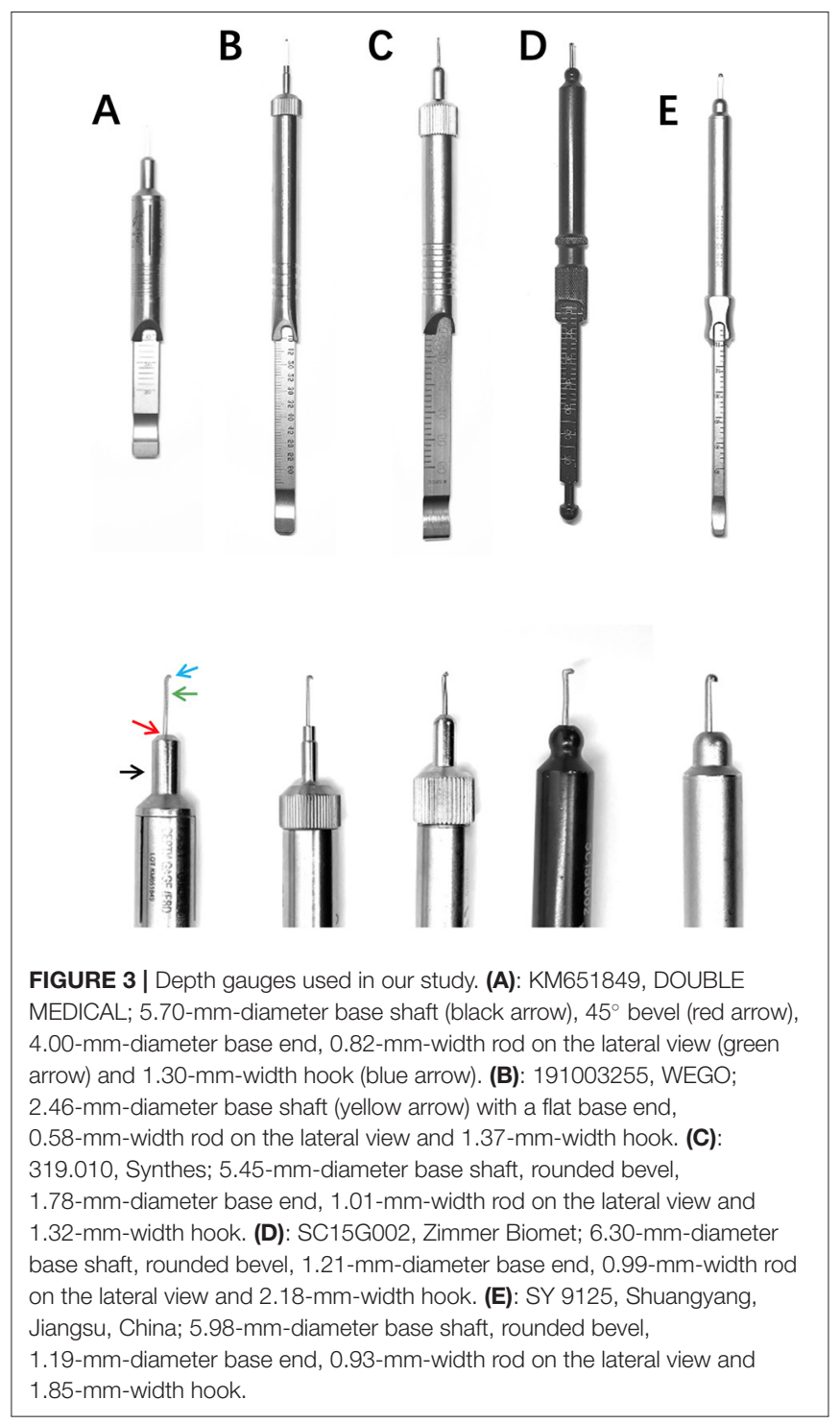

and scale from 0 to $150 \mathrm{~mm}$ (01130048,349-055, Hengliang, Shanghai, China). L1, L2, and L3 were compared within the same system.

\section{Statistical Analysis}

Chi-square tests were used to analyze differences in the accuracy of measurements between surgeons and passages. One-way ANOVA was used to compare the efficiency of measurements among the groups of surgeons. $P<0.05$ was considered statistically significant.

\section{RESULTS}

\section{Accuracy and Efficiency of Measurements Between Surgeons}

A total of 13 surgeons participated in 585 measurements and each surgeon completed 45 measurements (Figure 5). The 

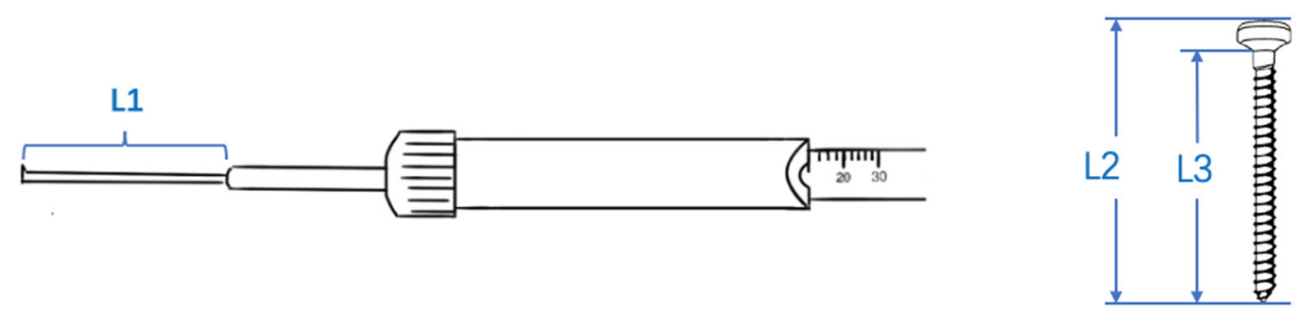

FIGURE 4 | Depth gauges from five manufacturers were placed on a $12 \mathrm{~mm}$ scale to measure the length of the rod (L1) and the length of $12 \mathrm{~mm}$ cortical bone screws [including its full length (L2) and length of the body part (L3)].

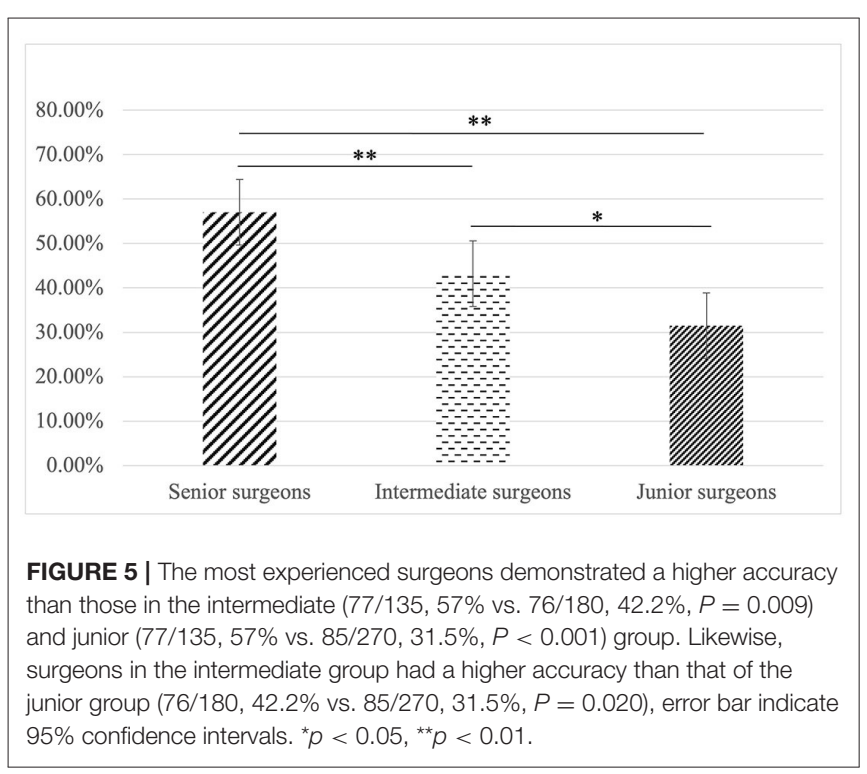

ideal screws were defined as a screw that reached the trans cortex but did not protrude more than $1 \mathrm{~mm}$ beyond it. Three senior surgeons completed 135 measurements, taking on average $689 \mathrm{~s}$ for 45 measurements with 77 ideal screws placed and an accuracy rate of $57.0 \%$. Four intermediate surgeons completed 180 measurements, taking on average $833 \mathrm{~s}$ for 45 measurements with 76 ideal length screws placed and an accuracy rate of $42.2 \%$. Finally, 6 junior surgeons completed 270 measurements, taking on average $785 \mathrm{~s}$ for 45 measurements with 85 ideal screws placed and an accuracy rate of $31.5 \%$.

The accuracy of measurements varied with the experience of surgeons $(p<0.001)$ (Table 1), in which the most experienced surgeons demonstrated a higher accuracy than those in the intermediate $(77 / 135,57 \%$ vs. $76 / 180,42.2 \%$, $P=0.009)$ and junior $(77 / 135,57 \%$ vs. $85 / 270,31.5 \%, P$ $<0.001$ ) group. Likewise, surgeons in the intermediate group had a higher accuracy than that of the junior group $(76 / 180,42.2 \%$ vs. $85 / 270,31.5 \%, P=0.020)$. Therefore, with more experience, the accuracy rate and efficiency of measurements using the depth gauge significantly improved $(P=0.033)$.
TABLE 1 | Accuracy and efficiency of measurements between surgeons.

\begin{tabular}{|c|c|c|c|c|}
\hline & Senior & Intermediate & Junior & $P$-value \\
\hline $\begin{array}{l}\text { Number of accurate } \\
\text { measurements }\end{array}$ & 77 & 76 & 85 & \\
\hline Accuracy rate & $\begin{array}{l}77 / 135 \\
(57.0 \%)\end{array}$ & $\begin{array}{l}76 / 180 \\
(42.2 \%)\end{array}$ & $\begin{array}{l}85 / 270 \\
(31.5 \%)\end{array}$ & $P<0.001$ \\
\hline $\begin{array}{l}\text { Number of accurate } \\
\text { measurements (seconds) }\end{array}$ & 689 & 833 & 785 & \\
\hline $\begin{array}{l}\text { Efficiency (number of } \\
\text { screws per minute) }\end{array}$ & 2.28 & 1.46 & 1.26 & $P=0.033$ \\
\hline
\end{tabular}

The accuracy and efficiency of measurements varied with the experience of surgeons $(p<0.001)$. Increased surgeon's experience was associated with improvements in the accuracy rate and measurement efficiency of drilled holes.

\section{Relationship Between the Accuracy Rate of Measurements and the Location and Passages of Drilled Holes}

Of the 585 measurements, 195 were made with straight drilling through the metaphysis, for which 88 screws were at an ideal length (accuracy rate of $45.1 \%$ ). For straight drilling through the diaphysis 85 screws were at an ideal length (accuracy rate of $43.6 \%$ ), whereas for angled drilling through the diaphysis, 65 screws were at an ideal length (accuracy rate of 33.3\%). Interestingly, the accuracy rate of measurements varied with the location and passage of the drilled holes $(P=0.036)$ but there was no statistical difference between straight metaphyseal and diaphyseal drilling $(85 / 195,43.6 \%$ vs. $88 / 195,45.1 \% ; P=0.760)$. Meanwhile, the accuracy of measurements was significantly lower for angled diaphyseal drilling when compared to that of straight diaphyseal drilling $(65 / 195,33.3 \%$ vs. $85 / 195,43.6 \%$; $P$ $=0.037)$ and straight metaphyseal drilling $(65 / 195,33.3 \%$ vs. $88 / 195,45.1 \% ; P=0.017$ ) (Figure 6).

\section{Measurement of Different Depth Gauges and Screws}

The results showed that $\mathrm{L} 1$ values were $10.30 \mathrm{~mm}$ (319.010, Synthes), $9.05 \mathrm{~mm}$ (Shuangyang, Jiangsu, China), $10.05 \mathrm{~mm}$ (SC15G002, Zimmer Biomet), $12.03 \mathrm{~mm}$ (KM651849, DOUBLE MEDICAL) and $9.30 \mathrm{~mm}$ (191003255, WEGO). 


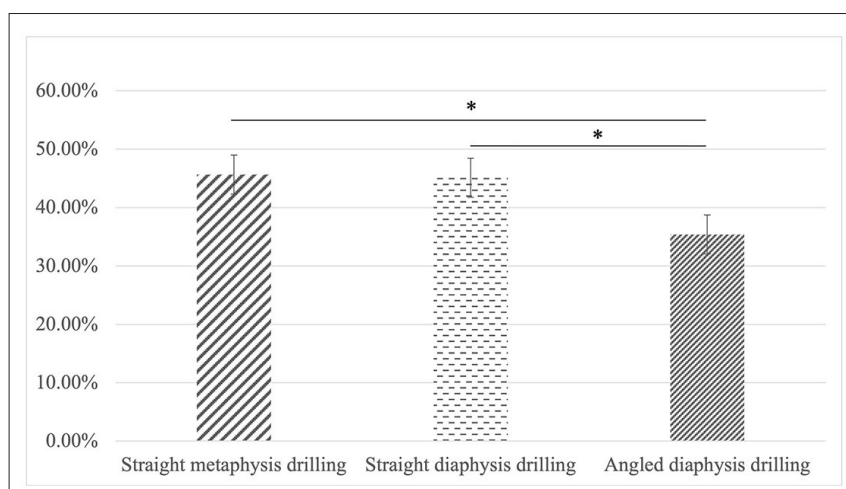

FIGURE 6 | There was no statistical difference between straight metaphyseal and diaphyseal drilling (85/195, 43.6\% vs. 88/195, 45.1\%; $P=0.760)$. Meanwhile, the accuracy of measurements was significantly lower for angled diaphyseal drilling when compared to that of straight diaphyseal drilling (65/195, 33.3\% vs. 85/195, 43.6\%; $P=0.037)$ and straight metaphyseal drilling $(65 / 195,33.3 \%$ vs. $88 / 195,45.1 \% ; P=0.017) .{ }^{*} p<0.05$.

TABLE 2 | Matching degree between depth gauge and screws from different manufacturers.

\begin{tabular}{lccc}
\hline Manufacturer & $\begin{array}{c}\text { Length of the } \\
\text { rod (L1, mm) }\end{array}$ & $\begin{array}{c}\text { Full length of the } \\
\text { screw (L2, mm) }\end{array}$ & $\begin{array}{c}\text { Length of the body } \\
\text { part (L3, mm) }\end{array}$ \\
\hline Synthes & 10.30 & 12.30 & 10.30 \\
Shuangyang & 9.05 & 12.00 & 10.40 \\
Zimmer biomet & 10.05 & 13.50 & 11.10 \\
Double medical & 12.03 & 12.00 & 10.45 \\
WEGO & 9.30 & 11.98 & 9.62 \\
\hline
\end{tabular}

Depth gauges from five manufacturers were placed on a $12 \mathrm{~mm}$ scale to measure the length of the rod $(L 1)$ and the length of $12 \mathrm{~mm}$ cortical bone screws [including its full length (L2) and length of the body part (L3)].

The L2 values were $12.30 \mathrm{~mm}$ (404.812, Synthes), $12.00 \mathrm{~mm}$ (0106936794823478, Shuangyang), $13.50 \mathrm{~mm}$ (815037012, Zimmer Biomet), $12.00 \mathrm{~mm}$ (040010022, DOUBLE MEDICAL) and $11.98 \mathrm{~mm}$ (6133512, WEGO). The L3 values were $10.30 \mathrm{~mm}$ (404.812, Synthes), $10.40 \mathrm{~mm}$ (0106936794823478, Shuangyang), $11.10 \mathrm{~mm}$ (815037012, Zimmer Biomet), $10.45 \mathrm{~mm}$ (040010022, DOUBLE MEDICAL) and $9.62 \mathrm{~mm}$ (6133512, WEGO) (Table 2).

\section{DISCUSSION}

This study explored both observer factor and objective factors (the passages of the drilled holes, depth gauge from different manufacturers) associated with measurement accuracy with vernier calipers used as a gold standard.

There were statistical differences in the accuracy rate of measurements among different groups of surgeons, with more experienced surgeons having more accurate measurements. However, there was no significant difference in the time taken for each surgeon to measure 45 holes.

To further compare surgeons' skills, we calculated the efficiency of measurements (i.e., the number of holes accurately measured using the depth gauge per minute). Considering both the speed and accuracy rate of measurements, our results showed that the measurement efficiency was positively correlated with surgeon training, indicating that theoretical study and simulated operations of a depth gauge may be helpful to improve the accuracy of measurements among the surgeons. Other studies have also shown that it was helpful for residents to master their surgical skills in advance either in the form of lectures or simulated operations (23-30).

Using a cadaveric model that simulates ORIF of proximal phalangeal fractures, Jernigan et al. (20) measured drilled holes using a depth gauge without fluoroscopy assistance and studied the relationship between the level of training and the rates of ideal screw length selection among the surgeons. Ideal screws were defined as a screw that reached the volar cortex but did not protrude more than $1 \mathrm{~mm}$ beyond it and the study results showed that for 18 participants and a total of 648 selected screws, there was no relationship between the rate of ideal screw selection and level of training. Attending surgeons were less likely to place short screws or screws protruding more than $1 \mathrm{~mm}$ beyond the volar cortex. These results are in contrast to our analysis possibly because they used the proximal phalanx that has a short average transverse diameter and thin soft tissue on the opposite side, thus tactile feedback would be more evident. Also, the surgeons were more familiar with the anatomy of the proximal phalanx, which may also affect the accuracy of measurements. We used porcine femurs with skin, which have a relatively large average transverse diameter and thicker soft tissue, and the surgeons were relatively unfamiliar with the anatomy, thus the accuracy of the measurements was only related to the skill of the surgeons. Taken together, the accuracy rate of measurements may vary for different bones when selecting an ideal screw using the depth gauge, hence, this needs to be explored in future studies.

Our study also found no statistical difference in the accuracy rate of measurements between straight holes drilled through the diaphysis or metaphysis. However, the accuracy rate was significantly lower for angled drillings through the diaphysis, which may be attributed to a different situation in the measurement when the tip of the depth gauge hooked to the obtuse or acute angle of the fracture. According to the trauma treatment principle of the Association for the Study of Internal Fixation (AO/ASIF), the tip of the depth gauge should be hooked to the obtuse angle of the fracture in angled drilling holes, which, unfortunately, is often ignored by surgeons during the operation. Demsey et al. (21) drilled holes in three different clinically relevant conditions: straight drilling through the diaphysis, angled drilling through the diaphysis, and straight drilling through the metaphysis using laser range-finding sensors in pig bones and laser range-finder-based prototypes for depth measurements. The results showed that the accuracy of the device was lowest for straight diaphyseal drilling but the same for angled diaphyseal and straight metaphyseal drillings, which is contrary to our findings. This discrepancy may have occurred because the prototype device is still in its experimental stage, so the accuracy and algorithm may not be optimal. 


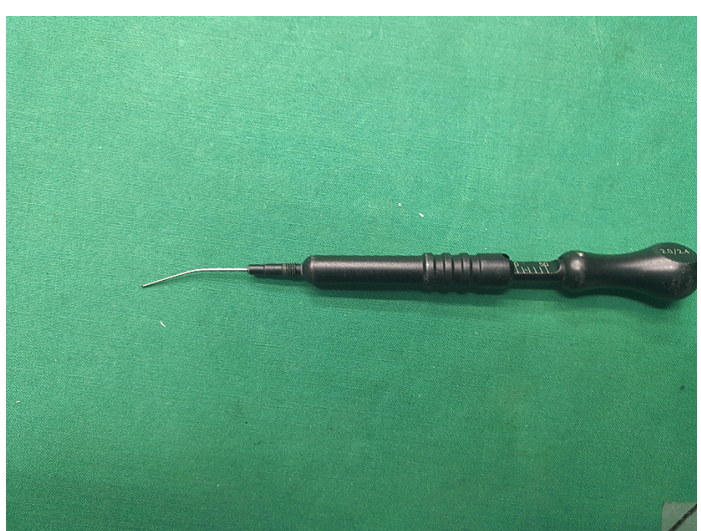

FIGURE 7 | The front probes of depth gauges may bend due to improper use, which may also lead to inaccurate measurements.

Also, laser displacement sensors were mounted on the surgical drill on either side of the drilling access in the horizontal plane and the laser gauge provided continuous displacement measurements relative to the surface illuminated by the laser line. The difference in distance estimates between the breach of the second cortex and the initial position was taken as the bore depth, therefore the accuracy differed in measuring the depth of drilled holes in bones compared with a conventional depth gauge.

Interestingly, depth gauges produced by different manufacturers yielded contrasting measuring values according to our measurements. The portion of the valid length, which is the body part of the screw, varied among different manufacturers and the actual length of the measurements using depth gauges were not strictly fit with the length of the 'selected screws' even within the same system. Moreover, the parameters of the depth gauges in our study varied with each other. McChesney et al. (22) mentioned that the amount of recess of the depth gauge base within a plate hole is influenced by the size and geometry of the depth gauge base and plate hole. A depth gauge with small-diameter base or long bevel may cause underestimate of the measurement. Further study could further investigate the influence of the geometry and width of the hook on the accuracy of measurement. A wide hook seems to have better feedback when measuring. Thus, surgeons should improve their understanding of the parameters of different manufacturers, systems, and screws in clinical practice to help in the selection of an ideal length screw based on a depth gauge.

The working state of the depth gauge may also affect the accuracy of the measurements. Rafique et al. (31) reported that an incorrect assembly of the depth gauge metal collar led to measurement errors. Also, the front probes of some depth gauges may bend due to improper use (Figure 7), which may also lead to inaccurate measurements. Therefore, before using the depth gauge, surgeons must check its state to avoid similar mechanical failures affecting the measurement accuracy.

Although our study explored effects related to the accuracy of measurements using a depth gauge, there were still some limitations. First, porcine femurs with skin rather than cadaver bone were used in this study, and their tactile feedback may be different. Porcine femurs are often used in teaching operations in orthopedics and are regarded as having similar components to the human bone $(21,26)$. In our study, we used porcine femurs with skin and kept the surrounding soft tissue to simulate a real surgical scene. Moreover, the screws were not truly screwed into the bones. Instead, the ideal length of the screw was determined only by the measurement using the depth gauge. However, in clinical practice, the surgeon can be assisted by the torsion force when the screw breaks through the trans cortex of the bone. It is crucial to determine whether the screw length is suitable when using the depth gauge for the first time as screw replacement may loosen the canal and decrease the screw grip. For an individual patient, the density of the bone, the type, and site of the fracture, as well as the diameter of the screw may all affect the holding force of the screw. According to Battula et al. (10), in normal bones, the depth of insertion of the tip of the screw should be $\sim 1 \mathrm{~mm}$ past the far cortex, while in osteoporotic bone, this should be at least $2 \mathrm{~mm}$ past the far cortex. However, Schoenfeld et al. (32) found that the biomechanical conclusions of selftapping screws simulated on artificial or corpse bone could not be applied to clinical practice, and for people with either a healthy bone or osteoporosis, the depth of insertion of the tip of the screw for adequate fracture fixation was at least $2 \mathrm{~mm}$ past the far cortex. In our study, the holding force and the protection of soft tissue were considered, therefore, the ideal screw was defined as a screw that reached the trans cortex but did not protrude more than $1 \mathrm{~mm}$, with a certain guiding significance in clinical practice.

\section{CONCLUSION}

Screw selection with appropriate length is crucial in orthopedic surgery. Based on our findings, both observer factor and objective factors could affect the accuracy of depth gauge measurement. Increased surgeon's experience was associated with improvements in the accuracy rate and measurement efficiency of drilled holes based on the depth gauge. Theoretical study and simulated operations of a depth gauge may be helpful to improve the accuracy of measurements among the surgeons. The accuracy rate varied with hole passages, being the lowest for angled drilled holes. Moreover, Parameters of depth gauges and screws varied among different manufacturers.

\section{DATA AVAILABILITY STATEMENT}

The raw data supporting the conclusions of this article will be made available by the authors, without undue reservation.

\section{ETHICS STATEMENT}

Ethical review and approval was not required for the animal study as purchased porcine femurs were used, which does not involve living animals. 


\section{AUTHOR CONTRIBUTIONS}

PL, JX, and XW: conception and design of the research. PL, JX, and CZ: writing of the manuscript. PL, CZ, and FY: acquisition of data. PL, CZ, XL, and GS: analysis and interpretation of the data and statistical analysis. XW: obtaining financing and critical revision of the manuscript for intellectual content. All authors contributed to the article and approved the submitted version.

\section{REFERENCES}

1. Endres K, Marx R, Tinschert J, Wirtz DC, Stoll C, Riediger D, et al. A new adhesive technique for internal fixation in midfacial surgery. Biomed Eng Online. (2008) 7:16. doi: 10.1186/1475-925X-7-16

2. Juvonen T, Nuutinen JP, Koistinen AP, Kroger H, Lappalainen R. Biomechanical evaluation of bone screw fixation with a novel bone cement. Biomed Eng Online. (2015) 14:74. doi: 10.1186/s12938-015-0069-6

3. Gholampour S, Deh HHH. The effect of spatial distances between holes and time delays between bone drillings based on examination of heat accumulation and risk of bone thermal necrosis. Biomed Eng Online. (2019) 18:65. doi: 10.1186/s12938-019-0686-6

4. Gholampour S, Hassanalideh HH, Gholampour M, Frim D. Thermal and physical damage in skull base drilling using gas cooling modes: FEM simulation and experimental evaluation. Comput Methods Programs Biomed. (2021) 212:106463. doi: 10.1016/j.cmpb.2021.106463

5. Gholampour S, Shakouri E, Deh HHH. Effect of drilling direction and depth on thermal necrosis during tibia drilling: an in vitro study. Technol Health Care. (2018) 26:687-97. doi: 10.3233/THC-181246

6. Jamil M, Khan AM, Mia M, Iqbal A, Gupta MK, Sen B. Evaluating the effect of micro-lubrication in orthopedic drilling. Proc Inst Mech Eng H. (2019) 233:1024-41. doi: 10.1177/0954411919865389

7. Shakouri E, Haghighi Hassanalideh H, Gholampour S. Experimental investigation of temperature rise in bone drilling with cooling: a comparison between modes of without cooling, internal gas cooling, and external liquid cooling. Proc Inst Mech Eng H. (2018) 232:45-53. doi: 10.1177/0954411917742944

8. Al-Rashid M, Theivendran K, Craigen MA. Delayed ruptures of the extensor tendon secondary to the use of volar locking compression plates for distal radial fractures. J Bone Joint Surg Br. (2006) 88:1610-2. doi: 10.1302/0301-620X.88B12.17696

9. Alajmo G, Schlegel U, Gueorguiev B, Matthys R, Gautier E. Plunging when drilling: effect of using blunt drill bits. J Orthop Trauma. (2012) 26:482-7. doi: 10.1097/BOT.0b013e3182336ec3

10. Battula S, Schoenfeld A, Vrabec G, Njus GO. Experimental evaluation of the holding power/stiffness of the self-tapping bone screws in normal and osteoporotic bone material. Clin Biomech. (2006) 21:533-7. doi: 10.1016/j.clinbiomech.2005.12.020

11. Clitherow HD, Bain GI. Association between screw prominence and vascular complications after clavicle fixation. Int J Shoulder Surg. (2014) 8:122-6. doi: 10.4103/0973-6042.145261

12. Clitherow HD, Bain GI. Major neurovascular complications of clavicle fracture surgery. Shoulder Elbow. (2015) 7:3-12. doi: 10.1177/1758573214546058

13. Johnson B, Thursby P. Subclavian artery injury caused by a screw in a clavicular compression plate. Cardiovasc Surg. (1996) 4:414-5. doi: 10.1016/0967-2109(95)00095-X

14. Karkos CD, Hughes R, Prasad V, D'Souza SP. Thigh compartment syndrome as a result of a false aneurysm of the profunda femoris artery complicating fixation of an intertrochanteric fracture. J Trauma. (1999) 47:393-5. doi: 10.1097/00005373-199908000-00033

15. Berkowitz R, Njus G, Vrabec G. Pullout strength of self-tapping screws inserted to different depths. J Orthop Trauma. (2005) 19:462-5. doi: 10.1097/01.bot.0000161544.72757.95

\section{FUNDING}

This work was supported by the National Natural Science Foundation of China (grant numbers 81871791 and 81272036).

\section{ACKNOWLEDGMENTS}

We are particularly grateful to all the people who have given us help on our article.

16. Marmor M, Mirick G, Matityahu A. Screw stripping after repeated cortical screw insertion-can we trust the cancellous "bailout" screw? J Orthop Trauma. (2016) 30:682-6. doi: 10.1097/BOT.0000000000000685

17. Matityahu A, Hurschler C, Badenhop M, Stukenborg-Colsman C, Waizy H, Wentz B, et al. Reduction of pullout strength caused by reinsertion of 3.5-mm cortical screws. J Orthop Trauma. (2013) 27:170-6. doi: 10.1097/BOT.0b013e31825490b1

18. Mejia A, Solitro G, Gonzalez E, Parekh A, Gonzalez M, Amirouche F. Pullout strength after multiple reinsertions in radial bone fixation. Hand. (2020) 15:393-8. doi: 10.1177/1558944718795510

19. Schoenfeld AJ, Battula S, Sahai V, Vrabec GA, Corman S, Burton L, et al. Pullout strength and load to failure properties of self-tapping cortical screws in synthetic and cadaveric environments representative of healthy and osteoporotic bone. J Trauma. (2008) 64:1302-7. doi: 10.1097/TA.0b013e318169cd71

20. Jernigan EW, 3rd, Honeycutt PB, Patterson JMM, Rummings Jr WA, Bynum $\mathrm{DK}$, et al. Accuracy in screw selection in a cadaveric, small-bone fracture model. J Hand Surg Am. (2018) 43:1138 e1-8. doi: 10.1016/j.jhsa.2018.04.011

21. Demsey D, Gomez Arrunategui JP, Carr NJ, Guy P, Hodgson AJ. Using laser range-finding to measure bore depth in surgical drilling of bone. Clin Orthop Relat Res. (2019) 477:2579-85. doi: 10.1097/CORR.00000000000 00922

22. McChesney D, Langenbach A, Kruger K, C Garcia T, Marcellin-Little DJ. Evaluation of depth gauge accuracy in a canine tibial plateau leveling osteotomy model. Vet Surg. (2021) 50:1389-97. doi: 10.1111/vsu. 13694

23. Homma Y, Mogami A, Baba T, Naito K, Watari T, Obayashi O, et al. Is actual surgical experience reflected in virtual reality simulation surgery for a femoral neck fracture? Eur J Orthop Surg Traumatol. (2019) 29:1429-34. doi: 10.1007/s00590-019-02465-9

24. Kim HJ, Kim DH, Kyung HS. Evaluation of arthroscopic training using a porcine knee model. J Orthop Surg. (2017) 25:2309499016684433. doi: $10.1177 / 2309499016684433$

25. LeBlanc J, Hutchison C, Hu Y, Donnon T. A comparison of orthopaedic resident performance on surgical fixation of an ulnar fracture using virtual reality and synthetic models. J Bone Joint Surg Am. (2013) 95:e60:S1-5. doi: 10.2106/JBJS.K.01284

26. Martin RK, Gillis D, Leiter J, Shantz JS, MacDonald P. A porcine knee model is valid for use in the evaluation of arthroscopic skills: a pilot study. Clin Orthop Relat Res. (2016) 474:965-70. doi: 10.1007/s11999-015-4498-0

27. Mayne IP, Brydges R, Moktar J, Murnaghan ML. Development and assessment of a distal radial fracture model as a clinical teaching tool. J Bone Joint Surg Am. (2016) 98:410-6. doi: 10.2106/JBJS.O.00565

28. Stirling ER, Lewis TL, Ferran NA. Surgical skills simulation in trauma and orthopaedic training. J Orthop Surg Res. (2014) 9:126. doi: 10.1186/s13018-014-0126-Z

29. Tarchala M, Charbonneau M, Aduljabbar FH, Teles AR, Weber M. Preeducation enhances the success of manual training for orthopedic surgery residents. J Surg Educ. (2019) 76:1433-9. doi: 10.1016/j.jsurg.2019.03.005

30. Yehyawi TM, Thomas TP, Ohrt GT, Marsh JL, Karam MD, Brown TD, et al. A simulation trainer for complex articular fracture surgery. J Bone Joint Surg Am. (2013) 95:e92. doi: 10.2106/JBJS.L.00554

31. Rafique B, Varghese J, Toft N. Don't be depth charged by the depth gauge. Arch Plast Surg. (2017) 44:356-7. doi: 10.5999/aps.2017.44.4.356 
32. Schoenfeld A, Vrabec G, Battula S, Salvator A, Njus G. Pullout strength variance among self-tapping screws inserted to different depths. Am J Orthop. (2008) 37:466-9.

Conflict of Interest: The authors declare that the research was conducted in the absence of any commercial or financial relationships that could be construed as a potential conflict of interest.

Publisher's Note: All claims expressed in this article are solely those of the authors and do not necessarily represent those of their affiliated organizations, or those of the publisher, the editors and the reviewers.
Any product that may be evaluated in this article, or claim that may be made by its manufacturer, is not guaranteed or endorsed by the publisher.

Copyright $\odot 2022$ Liu, Xiao, Zhao, Li, Sun, Yang and Wang. This is an open-access article distributed under the terms of the Creative Commons Attribution License (CC $B Y)$. The use, distribution or reproduction in other forums is permitted, provided the original author(s) and the copyright owner(s) are credited and that the original publication in this journal is cited, in accordance with accepted academic practice. No use, distribution or reproduction is permitted which does not comply with these terms. 